\title{
The disks and spheroid of LTGs in the light of their early web-like organization
}

\author{
R. Domínguez-Tenreiro ${ }^{1}$, A. Obreja ${ }^{1}$, C. Brook ${ }^{1}$, \\ F. J. Martínez-Serrano ${ }^{2}$ and A. Serna ${ }^{2}$ \\ ${ }^{1}$ Dept. de Física Teórica, Univ. Autónoma de Madrid, E-28049 Cantoblanco Madrid, Spain \\ ${ }^{2}$ Dept. de Física y A.C., Universidad Miguel Hernández, E-03202 Elche, Spain
}

\begin{abstract}
Cosmological hydrodynamical simulations show that the baryonic elements that at $z=0$ form the stellar populations of late-type galaxies (LTGs), display, at high $z$, a gaseous web-like organization, where different singular structures (walls, filaments, nodes) show up. The analysis also shows that the spheroid-to-be elements are the first to be involved in the singular structures, while thick and thin disk-to-be elements chronologically follow them. We discuss how these differences at high $z$ can explain the differences among these three components at $z=0$.
\end{abstract}

Keywords. cosmology: theory, galaxies: formation, methods: numerical

\section{Introduction}

The so-called "cold mode" for galaxy mass assembly was first formulated by Binney (1977) and (re)discovered in the last few years (e.g., Birnboim \& Dekel 2003; Kereš et al. 2003: Ocvirk et al. 2008; Brooks et al. 2009; Voort et al. 2011). This scenario has recently received much attention because it easily explains halo and galaxy angular momentum acquisition through filaments, (see e.g. Pichon et al. 2011 and this volume; Kimm et al. 2011; Codis et al. 2012; Tillson et al. 2012; Stewart et al. 2013; Dubois et al. 2014; Danovich et al. 2014) and also for its possible implications in the star formation history of galaxies ( Kereš et al. 2009; Brooks et al. 2009; Obreja et al. 2013).

On general grounds, cold streams feeding clumps is a general prediction of the Zeldovich Approximation (hereafter ZA, Zeldovich 1970) extended to the Adhesion Model (AM, see e.g. Gurbatov et al. 1989; 2012; Kofman et al. 1990), now confirmed in detail by numerical simulations (e.g., Cautun et al. 2014). Indeed, the AM predicts that, at a given scale, walls surrounding voids, filaments and nodes (i.e., the Cosmic Web, CW, elements, see Bond et al. 1996) are successively formed. Then they vanish due to mass elements flow through the voids towards the walls, through the walls towards the filaments, and finally from these to nodes, where mass elements pile up and virialize after turn-around and collapse (see e.g., Domínguez-Tenreiro et al. 2011). Meanwhile, the same CW elements emerge at larger and larger scales, disappearing later on in favour of voids and nodes.

We can conclude from these considerations that the stellar populations of current galaxies displayed at high $z$ a web-like structure. The question then arises whether or not current stellar populations in galaxies keep memory of their former lives as mass elements involved in the CW dynamics. More specifically, in this talk we focus on the origin of the so-called fine structure of late-type galaxies (hereafter, LTGs). Indeed, stellar populations of LTGs come into three different categories, those forming the spheroid, the thick disk and the thin disk, see Ivezić et al. (2012) and references therein. The differences among the components involve morphologies, stellar ages, velocity fields and chemical compositions, and are thought to be driven by their different specific angular momentum 


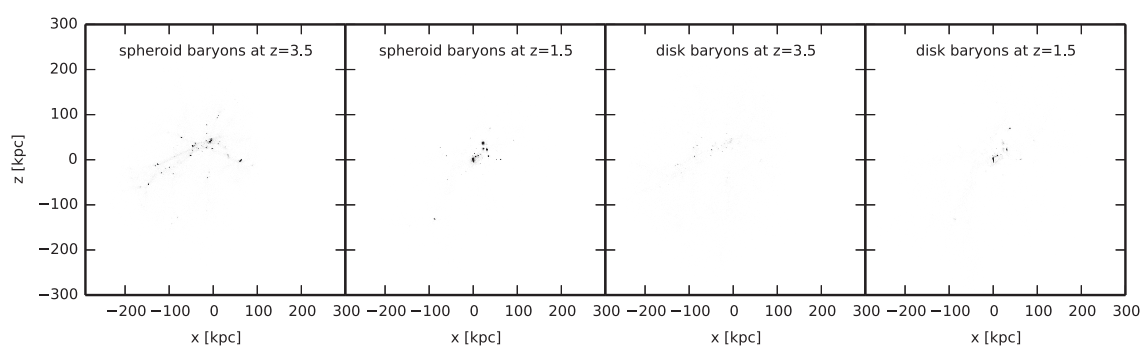

Figure 1. For the HD-5004A galaxy, a projection of the positions of its stellar particles identified at $z=0$ when traced back to $z_{\mathrm{high}}=3.5$ and 1.5. Left: spheroid-to-be particles. Right: thin disk-to-be particles. Grey (black): gaseous (stellar) particles at the respective $z_{\mathrm{high}} \mathrm{s}$.
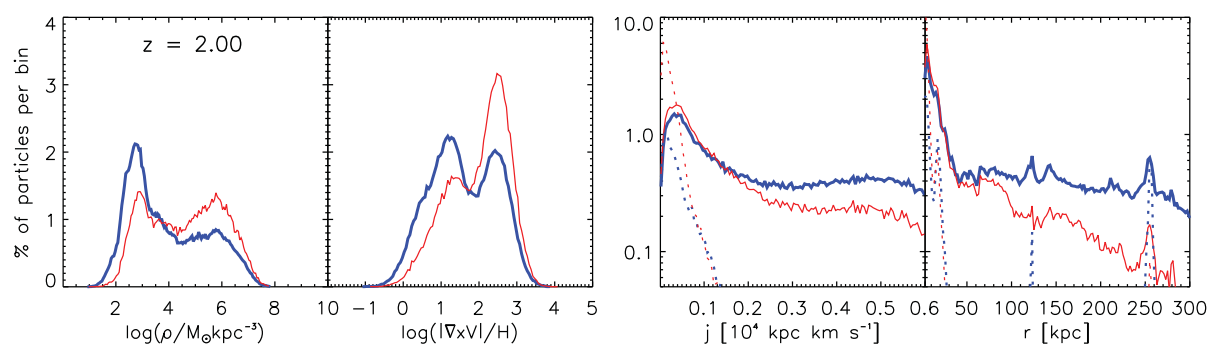

Figure 2. Histograms for different quantities measured on g1536- $\mathrm{L}^{*}$ galaxy -to-be particles. Solid red (thick blue) lines stand for gaseous spheroid (disk) precursors, while dashed lines refer to particles that are already stellar at $z=2$.

$j$ content, decreasing as we go from the thin disk to the spheroid components, with the thick disk in between.

Peebles (1969) and and Doroshkevich (1970) first pointed out that the $j_{L V}$ enclosed within a Lagrangian Volume grows as $a(t)^{2}$ untill turn around (i.e., the so-called Tidal Torques Theory, based on the ZA). Later on, $j_{L V}$ is roughly conserved, but redistributed within the LV from voids to filaments also as a consequence of the CW dynamics. Indeed, as first shown by Pichon el al. (2011), at the boundaries between voids, matter flows wind up coherently as they meet to form the filaments, causing whirls with their spins roughly parallel to their axes. At the same time, mass elements acquire a net transverse motion (i.e., an orbital $j$ relative to the patch center), due to the asymmetric forces acting on the forming filaments from the voids they divide. Once the filaments form, they collimate longitudinal motions towards the growing central mass accumulation, advecting $j$ in the process and coherently transporting it towards the inner central concentration, either as clumpy or diffuse material. Pichon et al. (2011) also show that, at a given time, the $j$ content of infalling matter grows with its distance to the patch center (lever effect). The implications this ordering has onto the $j$ acquisition by gas elements at the scales of galaxy halos have been analyzed recently by Stewart et al. (2013) and Danovich et al. (2014), who in particular have analyzed the gravitational torques responsible for gas and DM $j$ exchanges at the inner halo.

Hydrodynamical simulations in a cosmological context are an adequate tool to study the emergence of the fine-structure of LTGs. Comparisons among the results of different codes are more than advisable, because we are looking for effects coming from a generic and fundamental level of physical description, beyond the astrophysical scale. In view of these considerations, in this talk we present results of LTGs simulations run with two different SPH codes. In designing P-DEVA (Serna et al. 2003; Martínez-Serrano et al. 2008) the main concern was that $j$ conservation holds as accurately as possible. 
In GASOLINE (Wadsley et al. 2004), SNe feedback is implemented using the blastwaveformalism (Stinson et al. 2006), with a low effective coupling with gas. P-DEVA galaxies have been studied by Doménech et al. 2012 at $z=0$, who analyze some aspects of their fine structure, GASOLINE ones by Brook et al. (2012) and Obreja et al. (2014). In addition, Obreja et al. (2013) and Domínguez-Tenreiro et al. (2014) included galaxies of both sets. In all these cases, the consistency with observational data is very satisfactory.

To disentangle the fine structure of a given simulated LTG, Doménech et al. (2012) use the $k k$-means method, an unsupervised clustering algorithm looking for clusters in a 3-dimensional space of kinetic variables for each stellar particle of the LTG. By applying this method, with no priors assumed, the fine structure of LTGs emerges naturally (see Figure 7 in the former paper). Once classified, the systematics of spheroid, thick disk and thin disk observational properties is recovered (see also Obreja et al. 2013).

\section{Results and Conclusions}

To decipher the possible imprints left by the CW dynamics onto the current stellar populations of local LTGs, we have traced back these populations to their progenitors at high $z$. By plotting the positions of the progenitors of the stars that at $z=0$ form a LTG, we confirm that indeed they display a clear CW-like structure before collapse, whose evolution is as predicted by the AM. We also note that a main filament outstands in this structure (Dubois et al. 2014; Danovich et al. 2014) and that the disk is initially normal to this filament, as expected. Moreover, the systematics of $j$ acquisition by disks at the halo scale is recovered as well (see $\S 1$ ).

By separetely plotting the positions of the progenitors of each LTG component, we still found CW-like configurations, but with different characteristics. To illustrate these results, in Fig. 1 we plot, for a simulated LTG in the P-DEVA set, the projections on a given plane of the progenitor positions at $z_{\mathrm{high}}=3.5$ and 1.5 (collapse). The left panels correspond to the spheroid progenitors, while the right panels correspond to the thin disk antecessors. The visual impression is that spheroid progenitors have been involved in strong shell crossings from earlier on than thin disk progenitors, in such a way that at given $z$ s, they form a CW-like structure whose dynamical state is more advanced than that of the CW corresponding to the thin disk, with the thick disk in between.

To go a step further, the visual differences in Fig. 1 have been quantified by using the V-Web method (Hoffman et al. 2012). For a given simulated LTG and different $z_{\mathrm{high}} \mathrm{s}$, and for each of its gaseous or stellar particles, the densities, vorticities, $j_{i}$, and distances to the particles center-of-mass have been calculated, as well as the respective probability distributions for these quantities. The results we obtain confirm the visual impression that the spheroid-to-be gas particles are the first to be involved in shell-crossing events. To illustrate these results, in Fig. 2 we plot the respective histograms at $z_{\text {high }}=2$ for a GASOLINE galaxy. The histograms clearly show that, at given times, the spheroidto-be gas particles statistically have a higher probability to sample the highest densities and vorticities (marking the $\mathrm{CW}$ element locations). Moreover, the $j$ histograms confirm that the gas $j$ content increases as time goes by, caused by the more delayed gaseous particles having a higher probability of a bigger $j$ (lever effect, see $\S 1$ ). On the contrary, spheroid-to-be particles that by $z_{\text {high }}=2$ have already turned into stars are $j$-poorer. In addition, we also see that disk-to-be gaseous particles come on average from further away than spheroid-to-be ones, therefore the former are delayed relative to the later.

We conclude that these results obtained with two codes that are different in their design and subgrid modelling (see $\S 1$ ), strongly suggest that indeed the fine structure in LTGs expresses information imprinted in their baryonic mass elements at high $z$, when 
they display a CW configuration, and that this information comes from a fundamental level of physical description such as, e.g., the kinematics involved in the ZA or AM, see $\S 1$. In addition, our result that the local LTG fine structure (at disk scale) keeps memory of the CW dynamics at high $z$ suggests that, in the long run, the ordering it imprints in the cosmic inflow somehow overcomes the complex astrophysical processes occurring in the circumgalactic environment of forming disks.

We thank MICINN and MINECO (Spain) for financial support through grants AYA200912792-C03-02, -03 and AYA2012-31101 from the PNAyA.

\section{References}

Binney, J. 1977, ApJ, 215, 492

Birnboim, Y., Dekel A. 2003, MNRAS, 345, 349

Bond, J. R., Kofman, L., \& Pogosyan, D. 1996, Nature, 380, 603

Brook, C. B., Stinson, G. S., Gibson, B. K., Kawata, D., et al. 2012, MNRAS, 426, 690

Brooks, A. M., Governato, F., Quinn, T., Brook, C. B., \& Wadsley, J. 2009, ApJ, 694, 396

Cautun, M., van de Weygaert, R., Jones, B. J. T., \& Frenk, C. S. 2014, ArXiv e-prints

Codis, S., Pichon, C., Devriendt, J., Slyz, A., Pogosyan, D., Dubois, Y., \& Sousbie, T. 2012, MNRAS, 427, 3320

Danovich, M., Dekel, A., Hahn, O., Ceverino, D., \& Primack, J. 2014, ArXiv e-prints

Doménech-Moral, M., Martínez-Serrano, F. J., Domínguez-Tenreiro, R., \& Serna, A. 2012, MN$R A S, 421,2510$

Domínguez-Tenreiro, R., Oñorbe, J., Martínez-Serrano, F., \& Serna, A. 2011, MNRAS, 413, 3022

Domínguez-Tenreiro, R., Obreja, A., Granato, G. L., Schurer, A., et al. 2014, MNRAS 439, 3868

Doroshkevich, A. G. 1970, Afz, 6, 320

Dubois, Y., Pichon, C., Welker, C., Le Borgne, D., et al. 2014, MNRAS, 444, 1453

Gurbatov, S. N., Saichev, A. I., \& Shandarin, S. F. 1989, MNRAS, 236, 385 XYZ

Gurbatov, S. N., Saichev, A. I., \& Shandarin, S. F. 2012, Physics Uspekhi, 55, 223

Hoffman, Y., Metuki, O., Yepes, G., Gottlöber, S., Forero-Romero, J. E., Libeskind, N. I., \& Knebe, A. 2012, MNRAS, 425, 2049

Ivezić, Ž., Beers, T. C., \& Jurić, M. 2012, ARAA, 50, 251

Kereš, D., Katz, N., Fardal, M., Davé, R., Weinberg, D. H. 2009, MNRAS, 395, 160

Kereš, D., Katz, N., Weinberg, D. H., \& Davé, R. 2005, MNRAS, 363, 2

Kimm, T., Devriendt, J., Slyz, A., Pichon, C., Kassin, S. A., \& Dubois, Y. 2011, ArXiv e-prints

Kofman, L., Pogosian, D., \& Shandarin, S. 1990, MNRAS, 242, 200

Martínez-Serrano, F. J., Serna, A., Domínguez-Tenreiro, R., \& Mollá, M. 2008, MNRAS, 388, 39

Obreja, A., Brook, C. B., Stinson, G., Domínguez-Tenreiro, R., Gibson, B. K., Silva, L., \& Granato, G. L. 2014, MNRAS, 442, 1794

Obreja, A., Domínguez-Tenreiro, R., Brook, C., Martínez-Serrano, F. J., et al. 2013, ApJ, 763, 26

Ocvirk, P., Pichon, C., \& Teyssier, R. 2008, MNRAS, 390, 1326

Peebles, P. J. E. 1969, ApJ, 155, 393

Pichon, C., Pogosyan, D., Kimm, T., Slyz, A., Devriendt, J., \& Dubois, Y. 2011 MNRAS, 418, 2493

Serna, A., Domínguez-Tenreiro, R., \& Sáiz, A., 2003 ApJ, 597, 878

Stewart, K. R., Brooks, A. M., Bullock, J. S., Maller, A. H., Diemand, J., Wadsley, J., \& Moustakas, L. A. 2013, ApJ, 769, 74

Stinson, G., Seth, A., Katz, N., Wadsley, J., Governato, F., \& Quinn, T. 2006, MNRAS, 373, 1074

Tillson, H., Devriendt, J., Slyz, A., Miller, L., \& Pichon, C. 2012, ArXiv e-prints

van de Voort, F., Schaye, J., Booth, C., Haas, M., \& DallaVecchia, C. 2011, MNRAS, 414, 2458

Wadsley, J. W., Stadel, J., \& Quinn, T. 2004, New Astron., 9, 137

Zel'dovich, Y. B. 1970, $A \& A, 5,84$ 